\title{
Answer to the letter to the Editor of T. Smoljanovic et al. concerning "Posterior lumbar interbody fusion using rhBMP-2" [Meisel HJ et al. (2008), Eur Spine J 17:1735-1744]
}

\author{
H. J. Meisel
}

Published online: 8 April 2009

(C) Springer-Verlag 2009

I read your letter carefully and I want to answer selectively some of your concerns:

1. Paragraph 3, "As Meisel et al....": The authors are planning to undertake a dose-dependency analysis for a BMP2 in the fusion of vertebral bodies in a large animal model under GLP conditions. This study will start November 2009.

2. Paragraph 5: The authors state that the current lack of dose-dependency modeling in large animals and the conclusion drawn by Smoljanovic that transient vertebral resorption occurs regardless of the doses of BMP2 which have been used clinically is correct; further demonstrating that the available doses are still too high for intervertebral use.
3. Paragraph 6: All surgical interventions of the patients in the author's study received supplemental, rigid, posterior stabilization with pedicle screws at all levels integrated into the fusion. Due to this stabilization, no correction loss was detected during the observation time period of 2 years. Even though significant resorption of bone in the surrounding of the cages could be identified, cage migration was not observed.

In summary, a clear dose-dependency analysis for this treatment has to be undertaken before other clinical studies will follow. The underlying goal is to assure that the bone induction is sufficient, the construct is stable, and the clinical outcome meets the patient and physician expectations.

H. J. Meisel ( $\square)$

Department of Neurosurgery, BG-Clinic Bergmannstrost,

Merseburger Str. 165, 06112 Halle, Germany

e-mail: meisel@bergmannstrost.com 